\title{
Aggressive non-alcoholic steatohepatitis following rapid weight loss and/or malnutrition
}

\author{
Jia-Huei Tsai ${ }^{1,2}$, Linda D Ferrell ${ }^{3}$, Vivian Tan ${ }^{4}$, Matthew M Yeh ${ }^{5}$, Monika Sarkar ${ }^{6}$ and \\ Ryan M Gill ${ }^{3}$ \\ ${ }^{1}$ Department of Pathology, National Taiwan University Hospital, Taipei, Taiwan; ${ }^{2}$ Graduate Institute of \\ Pathology, College of Medicine, National Taiwan University, Taipei, Taiwan; ${ }^{3}$ Department of Pathology, \\ University of California, San Francisco, CA, USA; ${ }^{4}$ Genomic Health, Redwood City, CA, USA; ${ }^{5}$ Department of \\ Pathology, University of Washington, Seattle, WA, USA and ${ }^{6}$ Division of Gastroenterology and Hepatology, \\ Department of Medicine, University of California, San Francisco, CA, USA
}

\begin{abstract}
While non-alcoholic steatohepatitis is a slowly progressive disease, patients may rarely present in acute liver failure. We describe six patients who developed severe hepatic dysfunction following rapid weight loss or malnutrition. Rapid weight loss (18 to $91 \mathrm{~kg}$ ) occurred after Roux-en-Y gastric bypass in four patients and starvation-like dieting or hypoalbuminemia was noted in two patients. Four patients either died or received an urgent liver transplant. Pathologic findings were characterized by advanced alcoholic steatohepatitis-like features, including extensive/circumferential centrizonal pericellular fibrosis, central scar with perivenular sclerosis/veno-occlusion with superimposed hepatocellular dropout, abundant/prominent hepatocellular balloons, and numerous Mallory-Denk bodies, but there was no history of excess alcohol consumption. This study characterizes clinicopathologic features of aggressive non-alcoholic steatohepatitis following rapid weight loss or malnutrition, which should be included in the differential diagnosis with alcohol when a patient is considered for liver transplantation. The mechanism of liver injury in aggressive steatohepatitis is unknown, but rapid fat mobilization in obese patients may potentially cause oxidative stress to the liver and further study is needed to determine if there is a genetic predisposition to this form of injury and if antioxidants may protect the liver during rapid weight loss/malnutrition.
\end{abstract}

Modern Pathology (2017) 30, 834-842; doi:10.1038/modpathol.2017.13; published online 3 March 2017

The term 'non-alcoholic steatohepatitis' was first proposed by Ludwig et al. ${ }^{1}$ in 1980 to denote steatohepatitis in absence of alcohol abuse. Wellestablished risk factors for non-alcoholic steatohepatitis include obesity, metabolic syndrome, hyperlipidemia, and type 2 diabetes. ${ }^{2,3}$ Non-alcoholic steatohepatitis prevalence is increasing around the world, especially in the United States, ${ }^{4}$ and is expected to be the most common etiology of cirrhosis (and the most common indication for liver transplantation) in coming years. ${ }^{5}$ A multi-hit model has been proposed to explain the pathogenesis of nonalcoholic steatohepatitis. ${ }^{3}$ Proposed mechanisms of disease include proinflammatory cytokines, oxidative stress, and endoplasmic reticulum stress, as well

Correspondence: Dr RM Gill, MD, PhD, Department of Pathology, University of California San Francisco, 505 Parnassus Avenue, M545, Box 0102, San Francisco, CA 94143, USA.

E-mail: Ryan.Gill@ucsf.edu

Received 6 December 2016; revised 25 January 2017; accepted 26 January 2017; published online 3 March 2017 as an interplay between gut microbiome, diet, and genetic influences. ${ }^{3}$ Diagnosis of non-alcoholic steatohepatitis requires a liver biopsy with steatosis, lobular inflammation, and hepatocellular ballooning (with or without characteristic pericellular fibrosis). 6 ,7 Other common histologic findings in nonalcoholic steatohepatitis include Mallory-Denk bodies, acidophil bodies, glycogenosis, and glycogenated nuclei. Alcoholic steatohepatitis often manifests with more intense portal and lobular inflammation with neutrophil satellitosis, cholestasis, central vein obliteration, more prominent ballooned hepatocytes, and abundant Mallory-Denk bodies. ${ }^{6}$

Non-alcoholic steatohepatitis typically progresses slowly and may not be clinically apparent. ${ }^{2} \mathrm{Up}$ to $20 \%$ of patients with non-alcoholic steatohepatitis progress to cirrhosis over a 15-year time period. ${ }^{8}$ Hepatic decompensation in non-alcoholic steatohepatitis is rare and has been associated with drug use, rapid weight loss/malnutrition due to fasting, and intestinal resection or bariatric surgery. ${ }^{9-16}$ Rapid 
weight loss/malnutrition has been reported to induce hepatic inflammation and exacerbate steatohepatitis with progression to liver failure within a relatively short timeframe. ${ }^{17,18}$ However, liver injury due to rapid weight loss/malnutrition remains poorly understood and pathologic features have not been well-characterized. In this study, we describe six patients with rapid weight loss/malnutrition who developed severe hepatic dysfunction and histologic findings suggestive of an aggressive form of nonalcoholic steatohepatitis.

\section{Materials and methods}

\section{Case Selection}

To identify cases of potential aggressive nonalcoholic steatohepatitis, and following approval by the Committee on Human Research, the Department of Pathology, University of California, San Francisco (San Francisco, CA, USA) files were searched from 2000 to 2015 for 'steatohepatitis.' All cases suggestive of rapid progression/decompensation were selected for further review. Cases were excluded if there was any history of alcohol or drug abuse. We also received one case from the University of Washington Department of Pathology (Seattle, WA, USA). Demographic information is provided in Table 1.

All cases were evaluated by JHT, LDF, and RMG to document the following pathologic features: centrizonal scarring, perivenular sclerosis, centrizonal arteries, ${ }^{19}$ pericellular fibrosis, Mallory-Denk bodies, hepatocellular ballooning, ductular reaction, and cholestasis. Severe centrizonal scarring was defined as broad central-based fibrosis with extensive and circumferential pericellular fibrosis involving a majority of central zones. Trichrome and elastic stains (orcein stain or Elastic Van Gieson stain) were used to assess fibrosis and subacute hepatocellular dropout. ${ }^{20,21}$

\section{Results}

\section{Demographics and Clinical History}

All patients were female with a median age of 39 years (range 33-46 years; Table 1) and had a history of rapid weight loss or malnutrition. There was no history of prior liver disease. Patient 2 had detectable Epstein-Barr virus IgM, but clinical and histologic findings did not support acute Epstein-Barr virus hepatitis as a cause of liver failure. Five patients were morbidly obese; three underwent Roux-en-Y gastric bypass, one received long-limb Roux-en-Y gastric bypass, and one followed a low carbohydrate/ high protein diet for weight loss. There was no history of alcohol abuse or diet pill/supplement use. An intraoperative liver biopsy from Roux-en-Y gastric bypass was available in patient 1, which showed only moderate steatosis and early centrizonal pericellular fibrosis. The only non-obese patient in our study had hypoalbuminemia, suggestive of malnutrition, but no further history was available.

\section{Clinical Presentation and Outcome}

All patients presented with acute or subacute hepatic dysfunction, ranging from severe jaundice to asterixis, prominent hepatomegaly, and encephalopathy (with symptoms reported from 1 to 12 weeks prior to admission). There were two deaths and two patients received a liver transplant for severe liver failure soon after admission. The liver condition in one patient improved after medical management and one follow-up biopsy was taken three years later. Another patient had no available follow-up information.

\section{Pathologic Findings}

Pathologic findings are summarized in Table 2. Specimens included two explants (patients 2 and 3), two autopsies (patients 1 and 4), and two needle biopsies (patients 5 and 6). Massive hepatomegaly with fatty change was found in patients 1 and 4 , which weighed 6680 and $5600 \mathrm{~g}$, respectively. The other two liver explants, in patients 2 and 3 , showed micronodular cirrhosis and weighed 1825 and 1491g, respectively.

All histologic sections showed evidence of steatohepatitis, which was characterized by severe centralbased fibrous scars (Figure 1a and b), prominent hepatocellular ballooning, and abundant dense Mallory-Denk bodies (Figure 1c). There were apoptotic hepatocytes in lobular parenchyma and a prominent neutrophil infiltrate around ballooned hepatocytes in all cases. Centrizonal arteries were present to a variable extent in five patients (Figure 1d). There was focal ductular reaction, mostly in a periportal distribution, in five patients, while hepatocanalicular cholestasis was only identified in three patients (Figure 1e). Perivenular sclerosis was observed in five patients and was characterized by fibrous wall thickening, intimal fibrosis, or total venous sclerosis with luminal obliteration. (Figure 1f).

Trichrome stain was used to estimate extent of centrizonal scarring (Figure 2a) and perivenular sclerosis (Figure 2b). Most central zones showed severe fibrosis and were involved by broad fibrous scars with extensive and circumferential pericellular fibrosis throughout the centrizonal region (Zone 3), which also extended into Zone 2 (midzonal) and focally to the periportal region (Zone 1), with associated bridging fibrosis (ie, central-central bridge formation). Centrizonal two-tone trichrome staining intensity was evident in four patients (Figure 2c) with dense aggregates of thick collagen bundles in darker staining areas and, at most, thin collagen fibers dispersed loosely in lighter staining areas, 
Table 1 Patient characteristics and laboratory data on admission

\begin{tabular}{|c|c|c|c|c|c|c|}
\hline & Patient 1 & Patient 2 & Patient 3 & Patient 4 & Patient 5 & Patient 6 \\
\hline Age & 33 & 37 & 40 & 37 & 37 & 46 \\
\hline Gender & $\mathrm{F}$ & $\mathrm{F}$ & $\mathrm{F}$ & $\mathrm{F}$ & $\mathrm{F}$ & $\mathrm{F}$ \\
\hline Ethnicity & Hispanic & Caucasian & Hispanic & Caucasian & Caucasian & Caucasian \\
\hline Previous history & Morbid obesity & Morbid obesity & $\begin{array}{l}\text { Morbid obesity, anemia and } \\
\text { pancytopenia }\end{array}$ & Morbid obesity & Morbid obesity & $\begin{array}{l}\text { Previous history of fatty } \\
\text { liver and irregular menses }\end{array}$ \\
\hline Clinical setting & $\begin{array}{l}\text { Weight loss due to long-limb } \\
\text { Roux-en-Y bypass }\end{array}$ & $\begin{array}{l}\text { Weight loss due to Roux-en- } \\
\text { Y bypass }\end{array}$ & $\begin{array}{l}\text { Weight loss due to low } \\
\text { carbohydrate and high } \\
\text { protein diet }\end{array}$ & $\begin{array}{l}\text { Weight loss due to Roux-en-Y } \\
\text { bypass }\end{array}$ & $\begin{array}{l}\text { Weight loss due to Roux-en-Y } \\
\text { bypass }\end{array}$ & $\begin{array}{l}\text { 1-week history of } \\
\text { abdominal pain and } \\
\text { distention }\end{array}$ \\
\hline Alcohol & None & None & Occasionally & Occasionally & None & None \\
\hline Medications & None & Omeprazole & Omeprazole, vitamin $\mathrm{K}$ & Benadryl & None & None \\
\hline Weight loss/time & $18 \mathrm{~kg}$ over 5 months & $91 \mathrm{~kg}$ over 3 years & $23 \mathrm{~kg}$ over 1 year & $45 \mathrm{~kg}$ over 8 months & $40 \mathrm{~kg}$ over 7 years & Not available \\
\hline BMI changes & 45.3 to 38.4 & 59.4 to 26.5 & 39.7 to 30.2 & 55.1 to 36.5 & 46 to 32 & Current BMI: 24.5 \\
\hline Symptoms/signs & $\begin{array}{l}\text { Icteric skin, nausea, } \\
\text { vomiting, jaundice and } \\
\text { encephalopathy }\end{array}$ & $\begin{array}{l}\text { Jaundiced, asterixis, ascites, } \\
\text { prominent hepatomegaly }\end{array}$ & $\begin{array}{l}\text { Jaundiced, ascites and } \\
\text { encephalopathy }\end{array}$ & $\begin{array}{l}\text { Asterixis, icteric skin, prominent } \\
\text { hepatomegaly, lower extremity } \\
\text { edema, Right upper quadrant } \\
\text { abdominal pain }\end{array}$ & $\begin{array}{l}\text { Hepatomegaly, severe } \\
\text { malnutrition with peripheral } \\
\text { neuropathy and thiamine } \\
\text { deficiency, portal hypertension } \\
\text { with varices }\end{array}$ & Hepatomegaly \\
\hline $\begin{array}{l}\text { Initial symptoms } \\
\text { prior to admission }\end{array}$ & 19 days & 3 months & 2 months & Not available & Not available & 1 week \\
\hline \multirow{2}{*}{ Liver tests } & $\mathrm{AP}=189 \mathrm{U} / \mathrm{l}$ & & & $\begin{array}{l}\mathrm{ALT}=25 \mathrm{U} / 1 \\
\mathrm{AP}=98 \mathrm{U} / \mathrm{l}\end{array}$ & \multirow{2}{*}{$\begin{array}{l}\text { Transaminases: elevated } \\
\text { Bilirubin: within normal limits }\end{array}$} & $\begin{array}{l}\text { ALT }=22 \text { U/1 } \\
\text { AP: within normal limit }\end{array}$ \\
\hline & Total bilirubin $=11.7 \mathrm{mg} / \mathrm{dl}$ & Total bilirubin $=27.6 \mathrm{mg} / \mathrm{dl}$ & Total bilirubin $=35.2 \mathrm{mg} / \mathrm{dl}$ & Total bilirubin $=11 \mathrm{mg} / \mathrm{dl}$ & & Total bilirubin $=2.8 \mathrm{mg} / \mathrm{dl}$ \\
\hline Synthetic function & $\begin{array}{l}\text { Albumin }=2.4 \mathrm{~g} / \mathrm{dl} \\
\text { Evidence of coagulopathy }\end{array}$ & $\begin{array}{l}\text { Albumin }=1.8 \mathrm{~g} / \mathrm{dl} \\
\mathrm{INR}=2.4\end{array}$ & $\begin{array}{l}\text { Albumin }=2.1 \mathrm{~g} / \mathrm{dl} \\
\text { INR }=2.1\end{array}$ & $\begin{array}{l}\text { Albumin }=1.3 \mathrm{~g} / \mathrm{dl} \\
\text { INR }=1.4\end{array}$ & Evidence of coagulopathy & Albumin $=2.3 \mathrm{~g} / \mathrm{dl}$ \\
\hline \multirow{12}{*}{$\begin{array}{l}\text { Viral and } \\
\text { autoimmune } \\
\text { serologies }\end{array}$} & HAV IgM (-) & HAV IgM $(-)$ & HAV IgG (+) & HAV IgG (-) & \multirow[t]{12}{*}{ Not available } & \multirow{12}{*}{$\begin{array}{l}\text { HAV, HBV, HCV }(-) \\
\text { IgG = 1700, mildly } \\
\text { elevated } \\
\text { ANA(-) } \\
\text { LKM (-) }\end{array}$} \\
\hline & HBsAg $(-)$ & $\operatorname{HBsAg}(-)$ & $\operatorname{HAV} \operatorname{IgM}(-)$ & $\operatorname{HAV} \operatorname{IgM}(-)$ & & \\
\hline & $\operatorname{HBsAb}(-)$ & HBsAb $(-)$ & HBsAg $(-)$ & $\operatorname{HBsAg}(-)$ & & \\
\hline & HCV IgM $(-)$ & HBc IgM $(-)$ & HBcAb (-) & $\mathrm{HBcAb}(-)$ & & \\
\hline & CMV Ab (+) & $\operatorname{HCV} \operatorname{IgM}(-)$ & HBsAb $(+)$ & HBC IgM $(-)$ & & \\
\hline & ANA $<40$ & HIV Ab $(-)$ & HBc IgM $(-)$ & HCV Ab $(-)$ & & \\
\hline & & CMV Ab $(+)$ & HCV Ab $(-)$ & $\operatorname{HIV} \mathrm{Ab}(-)$ & & \\
\hline & & EBNA Ab $(+)$ & $\operatorname{HIV~Ab}(-)$ & CMV Ab $(-)$ & & \\
\hline & & EBV IgM (+) & CMV Ab (+) & EBNA Ab $(-)$ & & \\
\hline & & ANA $<40$ & EBNA Ab $(+)$ & $\operatorname{EBV} \operatorname{IgM}(-)$ & & \\
\hline & & Anti-smooth muscle (-) & EBV IgM $(-)$ & AMA $(-)$ & & \\
\hline & & LKM Ab (-) & $\begin{array}{l}\text { ANA }<40 \\
\text { AMA }(-)\end{array}$ & & & \\
\hline \multirow[t]{2}{*}{ Other labs } & $\begin{array}{l}\text { Ceruloplasmin: within } \\
\text { normal limits }\end{array}$ & $\begin{array}{l}\text { Ceruloplasmin: within } \\
\text { normal limits }\end{array}$ & $\begin{array}{l}\text { Ceruloplasmin: within } \\
\text { normal limits }\end{array}$ & $\begin{array}{l}\text { Ceruloplasmin: within normal } \\
\text { limits }\end{array}$ & & $\begin{array}{l}\text { Ceruloplasmin: within } \\
\text { normal limits }\end{array}$ \\
\hline & $\begin{array}{l}\text { Ferritin }=305 \mu \mathrm{g} / \mathrm{l} \\
\alpha \text {-1-antitrypsin: within } \\
\text { normal limits }\end{array}$ & $\begin{array}{l}\alpha \text {-1-antitrypsin: within } \\
\text { normal limits }\end{array}$ & $\begin{array}{l}\alpha \text {-1-antitrypsin: within } \\
\text { normal limits }\end{array}$ & Ferritin $=735 \mu \mathrm{g} / \mathrm{l}$ & & $\begin{array}{l}\alpha \text {-1-antitrypsin: within } \\
\text { normal limits }\end{array}$ \\
\hline Outcome & Death & Liver transplantation & Liver transplantation & Death & $\begin{array}{l}\text { Improved after medical } \\
\text { treatment }\end{array}$ & Not available \\
\hline
\end{tabular}

Abbreviations: Albumin (normal adult: 3.5-4.8 g/dl); ALT, alanine aminotransferase (normal adult female: 11-50 U/l); AMA, anti-mitochondrial antibody; ceruloplasmin (normal: 19-68 mg/dl); ANA, antinuclear antibody (normal: negative titer < 40); $\alpha$-1-antitrypsin (normal: 79-207 mg/dl); AP, alkaline phosphatase (normal: adult female: $31-95$ U/l); AST, aspartate aminotransferase (normal adult: 17-42 U/l); total bilirubin (normal: 0.2-1.3 mg/dl); BMI, body mass index; CMV, cytomegalovirus; EBNA, Epstein-Barr Virus nuclear antigen; EBV, Epstein-Barr virus; ferritin (normal female age 30-39: 12-160 $\mu \mathrm{g} / \mathrm{l}$ ); HAV, hepatitis A virus; HBsAg, hepatitis B surface antigen; HBsAb, hepatitis B surface antibody; HBc, hepatitis B core; HCV, hepatitis C; HIV, human immunodeficiency virus; INR, international normalized ratio (normal: 0.9-1.3); LKM Ab, liver kidney microsomal antibody. 


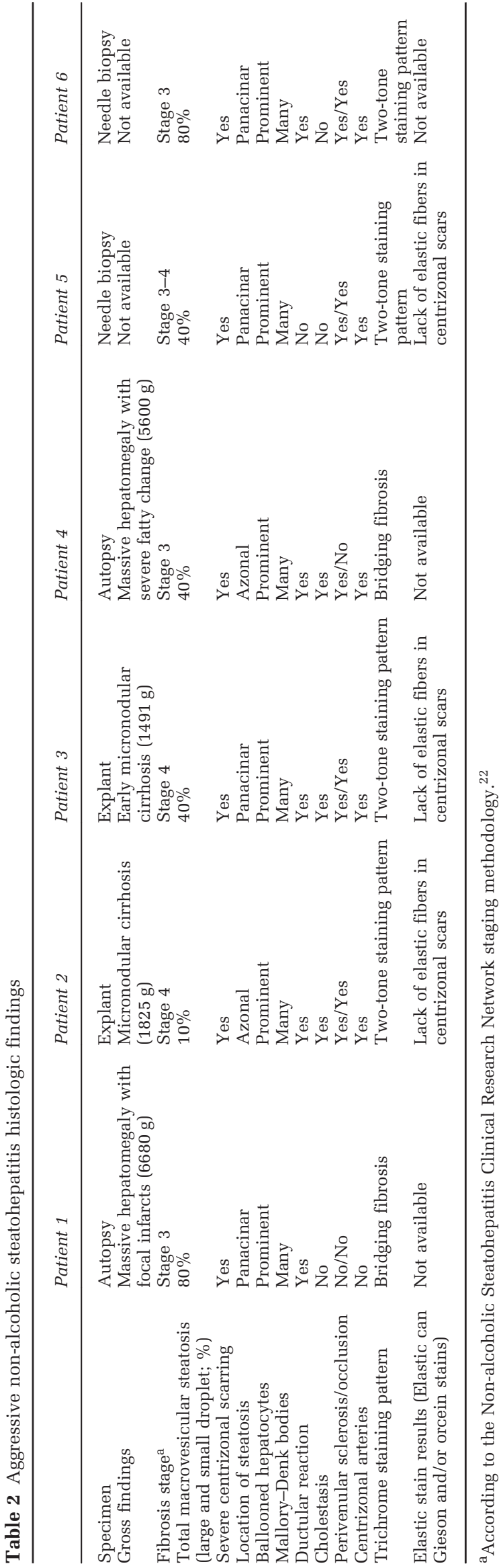

which suggested subacute injury. Elastic stains were performed on biopsies from three patients and demonstrated a lack of well-defined elastic fibers in pale staining areas, which confirmed our impression of subacute dropout rather than established fibrosis (Figure 2d).

Fibrosis was staged (summarized in Table 2) using Non-alcoholic Steatohepatitis Clinical Research Network methodology. ${ }^{22}$ In patient 5 , there was severe centrizonal scarring (as well as pale staining areas on trichrome stain suggestive of subacute dropout), sufficient for classification as at least bridging fibrosis (Figure 3a and b), which was overall considered stage 3-4 based on degree of pericellular fibrosis (Figure 3c). A follow-up biopsy was available three years after clinical resolution of liver failure, which demonstrated significant reduction in fibrosis with only few thin, bridging, collagenous bands around vaguely nodular hepatic lobules (Figure 3d), to suggest remodeling of prior fibrosis. Notably, no steatosis, ballooned hepatocytes, or Mallory-Denk bodies were seen in this biopsy.

\section{Discussion}

In this study, we describe a series of patients with an aggressive form of non-alcoholic steatohepatitis related to rapid weight loss/malnutrition, who had no significant alcohol exposure, and who developed severe liver dysfunction that usually resulted in liver transplantation or death. Liver biopsy demonstrated a characteristic combination of severe centrizonal scaring, abundant hepatocellular ballooning, and numerous Mallory-Denk bodies. Extensive pericellular fibrosis was the most striking finding and was well beyond what is typically seen in non-alcoholic steatohepatitis. Ductular reaction and cholestasis were also particularly prominent in three patients with elevated bilirubin. Aggressive non-alcoholic steatohepatitis shares similar histologic features with alcoholic steatohepatitis, namely abundant MalloryDenk bodies, prominent neutrophil infiltrate around ballooned hepatocytes, central vein obliteration, pericellular fibrosis, and cholestasis. ${ }^{6,7}$ The finding of subacute injury, which can be best identified on a well-controlled trichrome stain (though occasionally an elastic stain is needed for confirmation of these findings, as elastic fiber deposition supports early fibrosis ${ }^{20,21}$ ), further supports consideration of a rapidly progressive or acute/subacute process. Four of six patients had an increased aspartate aminotransferase/alanine aminotransferase ratio ( $>2: 1)$, which has been associated with alcoholic steatohepatitis. Although it is not possible to absolutely exclude concealment of significant alcohol consumption, increased aspartate aminotransferase/alanine aminotransferase ratio is not entirely specific for alcoholic steatohepatitis and has been described more generally with advanced fibrosis; ${ }^{23}$ also, specificity of this ratio has not been evaluated in rapidly 

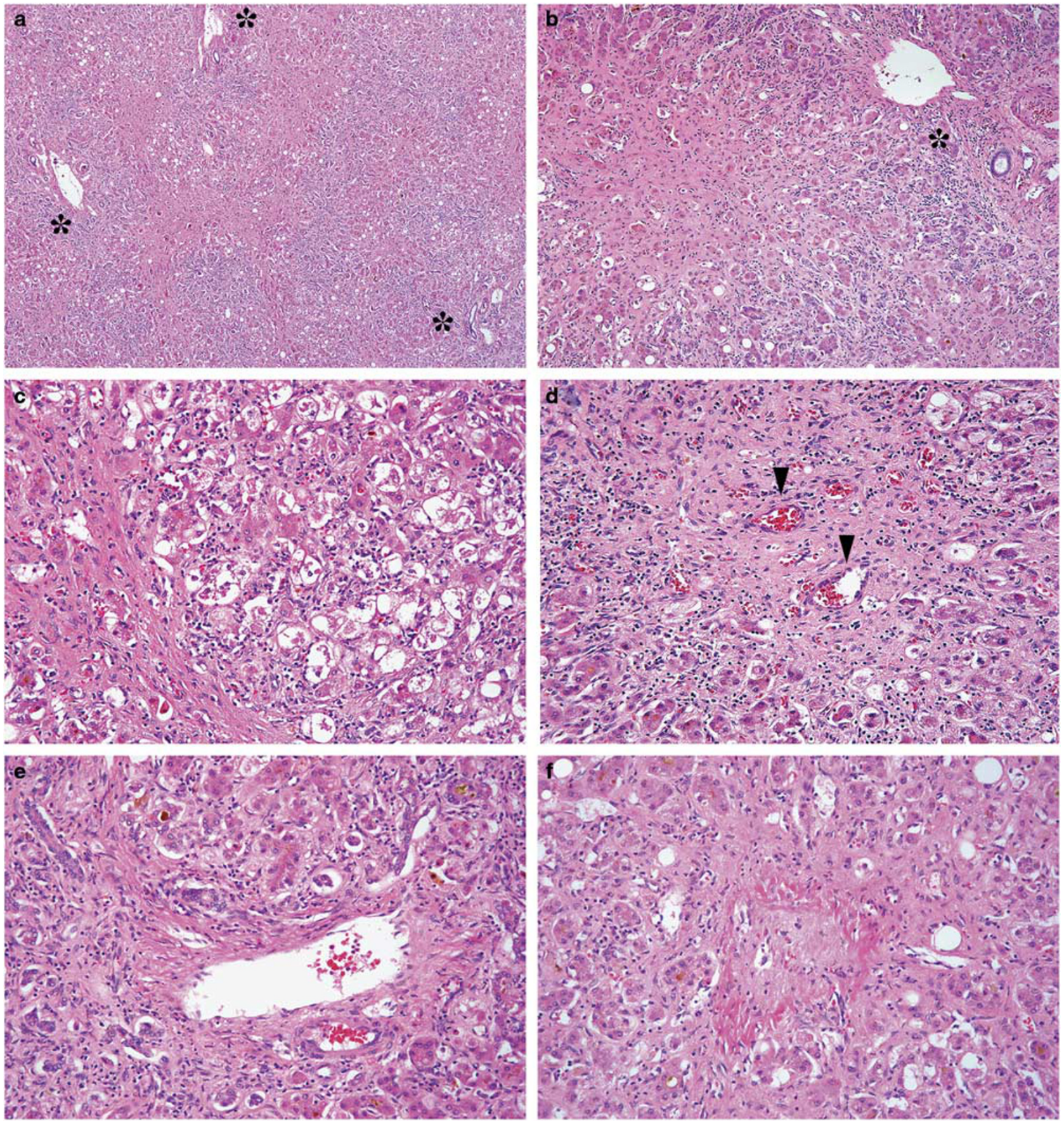

Figure 1 Pathologic features of aggressive non-alcoholic steatohepatitis in patient 2. (a) and (b) Examples of severe centrizonal scarring with pericellular fibrosis. Asterisks indicate portal tracts. (c) Prominent ballooned hepatocytes with Mallory-Denk bodies. (d) Centrizonal arteries (arrowheads) in fibrous central zones. (e) Ductular reaction and cholestasis. (f) Perivenular sclerosis with central vein occlusion. (a-f: H\&E stain; original magnification: $(\mathbf{a}): \times 40 ;(\mathbf{b}): \times 100 ;(\mathbf{c}-\mathbf{f}): \times 200)$. H\&E, hematoxylin and eosin.

progressive steatohepatitis. In our opinion, both alcoholic steatohepatitis and aggressive nonalcoholic steatohepatitis should be included in the histologic differential diagnosis when a patient presents with a rapidly progressive form of steatohepatitis.

It is estimated that $16 \%$ of bariatric surgery patients have incidental non-alcoholic steatohepatitis with nearly $4 \%$ expected to have bridging fibrosis or cirrhosis on initial evaluation, ${ }^{24}$ which suggests slow evolution of a clinically silent disease in this population. In contrast, aggressive non-alcoholic steatohepatitis presents with rapid liver failure before there is evidence of cirrhosis. This scenario was confirmed on liver biopsy from one patient in our study, who had only mild centrizonal pericellular fibrosis on initial biopsy, but after rapid weight loss developed aggressive non-alcoholic 

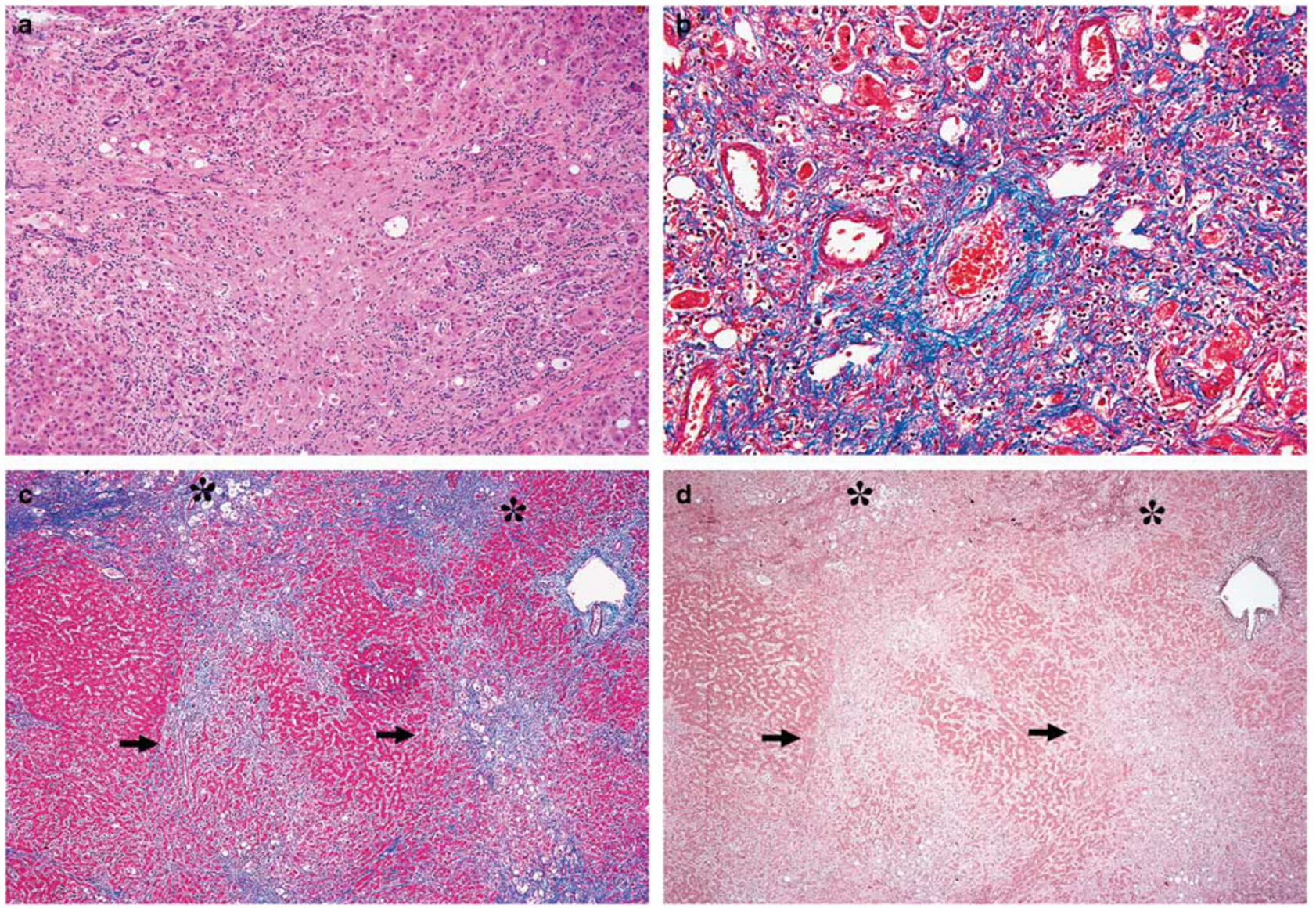

Figure 2 Additional examples of fibrosis in aggressive non-alcoholic steatohepatitis. (a) Broad dense centrizonal fibrosis, H\&E stain. (b) Perivenular sclerosis and intimal thickening. Centrizonal arteries are also present to the left of the vein, trichrome stain. (c) Subacute changes with two-tone trichrome staining; dense, thick collagen bundles stain dark blue (asterisks), while regions with subacute dropout stain pale blue (arrows), trichrome stain. (d) Elastic fiber deposition in early fibrosis (arrows-lack of elastic fiber deposition in regions of necrosis; asterisks—elastic fiber deposition in early fibrosis), orcein stain. (Original magnification: $(\mathbf{a}): \times 100 ; \mathrm{B}: \times 200 ;(\mathbf{c}, \mathbf{d}): \times 40)$. H\&E, hematoxylin and eosin.

steatohepatitis. These observations suggest an acute/ subacute mechanism of centrizonal liver injury related to rapid weight loss and/or malnutrition. Also, one survivor was found to have resolution of hepatocellular ballooning, with fibrosis remodeling, three years after presentation, to suggest that the liver may return to adequate function after weight stabilizes and/or nutrition returns to normal. Utilization of traditional staging and grading schemes for non-alcoholic steatohepatitis may not be appropriate in the setting of aggressive non-alcoholic steatohepatitis, as the original schemes were not developed to assess rapidly progressive liver injury. ${ }^{22}$

A mechanism for aggressive non-alcoholic steatohepatitis in the setting of rapid weight loss/malnutrition is not yet apparent. Since most affected patients, in other reports on rapid weight loss, were morbidly obese, attention has been drawn to an increase in lipolysis and enhanced release of endogenous free fatty acid from adipose deposits. ${ }^{18,25,26}$ Fat is rapidly mobilized from visceral adipose tissue and free fatty acids flood the portal circulation in rapid weight loss and may perhaps overwhelm hepatic parenchyma. ${ }^{25}$ Oxidation of free fatty acid by hepatocytes, which first occurs in the central zone, creates reactive oxygen species that may trigger mitochondrial injury and cytokine release (eg, tumor necrosis factor- $\alpha$ and interleukin-6), ${ }^{27}$ which may correspond to damage in Zone 3 (as with other agents known to increase liver free radical formation (eg, carbon tetrachloride, acetaminophen, ethanol) or in ischemia-reperfusion injury). Also, increased intestinal permeability in malnutrition leads to an influx of endotoxin into the portal circulation, which increases tumor necrosis factor- $\alpha$ production. ${ }^{28,29}$ Tumor necrosis factor- $\alpha$ has been specifically implicated as a biomarker in nonalcoholic steatohepatitis. ${ }^{30}$ Both fatty acids and endotoxin are hypothesized to aggravate the inflammatory activity of steatohepatitis. ${ }^{27}$ Furthermore, a fatty liver is less tolerant to oxidative stress than normal liver. ${ }^{18,31,32}$ Malnutrition also depresses hepatic antioxidant reserves and impairs overall metabolic function of mitochondria. ${ }^{33,34}$ Similarly, 

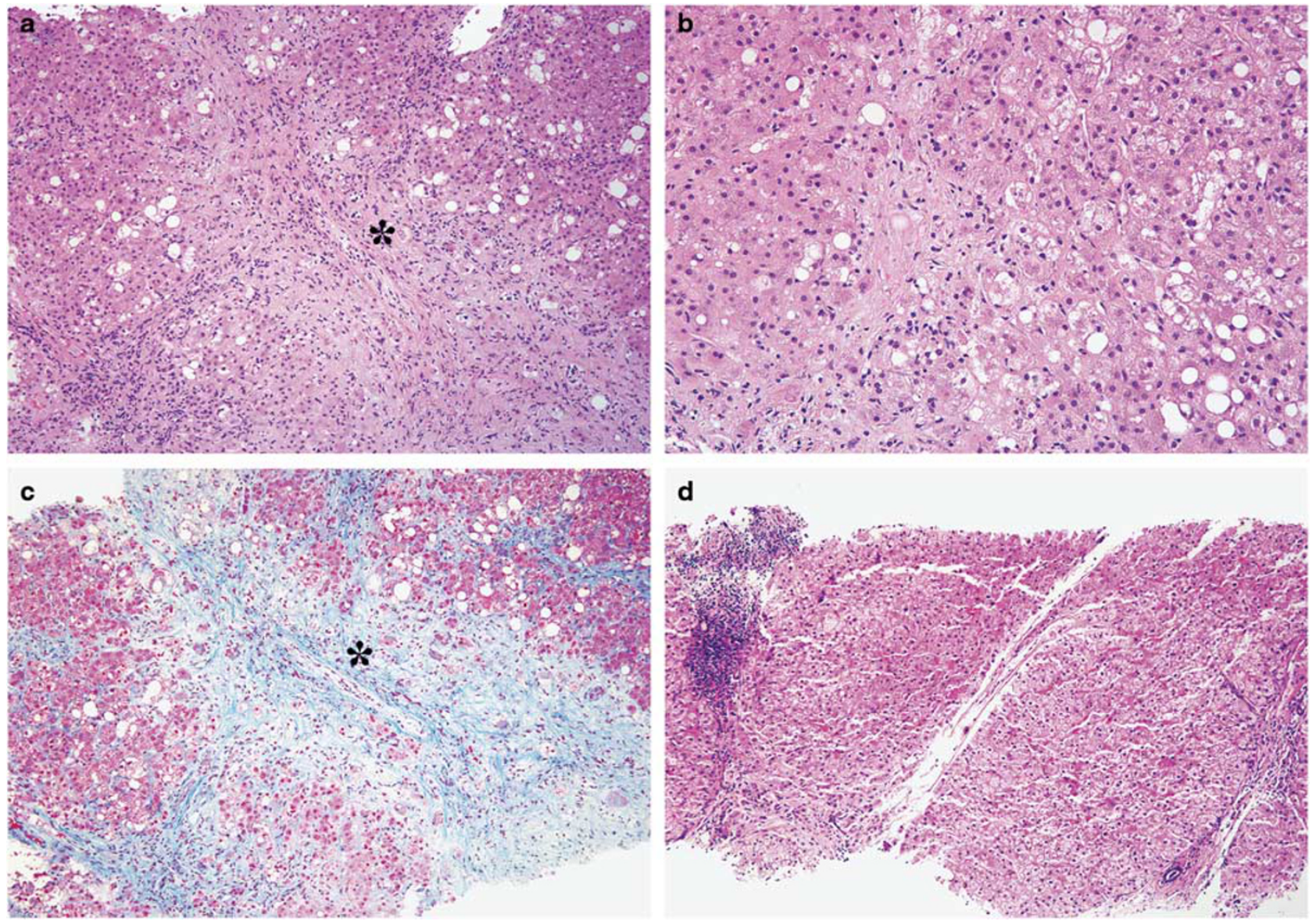

Figure 3 Histologic resolution/regression in patient 5. (a) Initial presenting biopsy at the time of liver failure with severe centrizonal scarring with central vein occlusion (asterisk), H\&E stain. (b) Initial biopsy with many ballooned hepatocytes and Mallory-Denk bodies, H\&E stain. (c) Initial biopsy with two-tone trichrome staining, highlighting centrizonal scar and obliterated vein (asterisk), trichrome stain. (d) Follow-up biopsy, three years after clinical resolution, without steatosis or ballooned hepatocytes. There are thin fibrous septa stretching from the central zones with mild/moderate portal and lobular inflammation, H\&E stain. (Original magnification: (a): $\times 100 ;(\mathbf{b})$ : $\times 200 ;(\mathbf{c}, \mathbf{d}): \times 100)$. H\&E, hematoxylin and eosin.

primary graft dysfunction, which can occur in association with markedly steatotic liver allografts, corresponds to an impaired ability to recover from ischemia reperfusion. ${ }^{35}$ It seems plausible that sustained delivery of enough free fatty acid (and perhaps associated endotoxin/cytokines), to an already compromised fatty liver, may underlie development of aggressive non-alcoholic steatohepatitis, ${ }^{18,31}$ but mechanistic studies are needed to further investigate this hypothesis and to consider a potential role for antioxidants to protect the liver during rapid weight loss/malnutrition. A genetic predisposition to developing rapidly progressive steatohepatitis is also a consideration and molecular testing could allow for additional insight into the mechanism of disease. For example, genome wide association analyses have identified specific gene polymorphisms (ie, patatin-like phospholipase domain containing 3 (PNPLA3, rs738409, encoding I148M) and transmembrane 6 superfamily member 2 (TM6SF2, rs58542926, encoding Glu167Lys)) that may confer increased susceptibility to development of steatohepatitis. ${ }^{3,36}$

Jejuno-ileal bypass is contraindicated for weight loss due to a high incidence of progressive hepatic disease leading to liver failure and cirrhosis. ${ }^{14}$ Such cases are reported to show histologic features similar to alcoholic steatohepatitis. ${ }^{37}$ Liver complications in jejuno-ileal bypass are complex, with only a subset of patients developing liver failure, and the etiology is considered multifactorial, with ongoing chronic liver injury noted in some patients. ${ }^{14,37,38}$ In addition to marked weight loss and severe malnutrition (related to poor absorption and severe diarrhea), the excluded intestine in this procedure is predisposed to bacterial overgrowth and synthesis of harmful endotoxin/lipopolysaccharide and/or bile acid metabolites. ${ }^{14,18,25,26,38}$ In contrast, Roux-en-Y gastric bypass has a shorter bypassed segment, with exposure to bile and pancreatic juice, which reduces bacterial overgrowth. ${ }^{39}$ In addition, Roux-en-Y gastric bypass usually achieves more controlled weight loss than jejuno-ileal bypass. ${ }^{17,26,40}$ Liver failure 
associated with Roux-en-Y gastric bypass is considered a rare event ${ }^{41}$ and cases of aggressive nonalcoholic steatohepatitis seen in our series were specifically associated with rapid weight loss and/or malnutrition rather than a surgical complication. Roux-en-Y gastric bypass is considered a safe procedure if drastic weight loss is avoided in the postoperative course. ${ }^{17,25}$

In conclusion, we have encountered several cases of aggressive non-alcoholic steatohepatitis in association with rapid weight loss/malnutrition, which can result in over-emphasis of a potential alcohol etiology in pathology reports. While it may not be possible to entirely exclude an alcohol etiology, acute liver failure with extensive pericellular fibrosis, steatosis, hepatocellular ballooning, MalloryDenk bodies, cholestasis, and central vein obliteration should not be specifically ascribed to alcohol and may represent an acute to subacute response to central zone injury during rapid weight loss/malnutrition. The histologic findings in aggressive nonalcoholic steatohepatitis may point to a similar mechanism of disease between alcoholic steatohepatitis and non-alcoholic steatohepatitis.

\section{Disclosure/conflict of interest}

The authors declare no conflict of interest.

\section{References}

1 Ludwig J, Viggiano TR, McGill DB, et al. Nonalcoholic steatohepatitis: Mayo Clinic experiences with a hitherto unnamed disease. Mayo Clin Proc 1980;55:434-438.

2 Farrell GC, Larter CZ. Nonalcoholic fatty liver disease: from steatosis to cirrhosis. Hepatology 2006;43:S99-S112.

$3 \mathrm{Yu}$ J, Marsh S, Hu J, et al. The Pathogenesis of Nonalcoholic Fatty Liver Disease: Interplay between Diet, Gut Microbiota, and Genetic Background. Gastroenterol Res Pract 2016;2016:2862173.

4 Younossi ZM, Koenig AB, Abdelatif D, et al. Global epidemiology of nonalcoholic fatty liver disease-Metaanalytic assessment of prevalence, incidence, and outcomes. Hepatology 2016;64:73-84.

5 Charlton MR, Burns JM, Pedersen RA, et al. Frequency and outcomes of liver transplantation for nonalcoholic steatohepatitis in the United States. Gastroenterology 2011;141:1249-1253.

6 Yeh MM, Brunt EM. Pathological features of fatty liver disease. Gastroenterology 2014;147:754-764.

7 Yeh MM, Brunt EM. Pathology of nonalcoholic fatty liver disease. Am J Clin Pathol 2007;128:837-847.

8 Angulo P. Long-term mortality in nonalcoholic fatty liver disease: is liver histology of any prognostic significance? Hepatology 2010;51:373-375.

9 Geerts A, Darius T, Chapelle T, et al. The multicenter Belgian survey on liver transplantation for hepatocellular failure after bariatric surgery. Transplant Proc 2010;42:4395-4398.

10 Nanki T, Koike R, Miyasaka N. Subacute severe steatohepatitis during prednisolone therapy for systemic lupus erythematosis. Am J Gastroenterol 1999;94:3379.

11 Capron JP, Delamarre J, Dupas JL, et al. Fasting in obesity: another cause of liver injury with alcoholic hyaline? Dig Dis Sci 1982;27:265-268.

12 Peura DA, Stromeyer FW, Johnson LF. Liver injury with alcoholic hyaline after intestinal resection. Gastroenterology 1980;79:128-130.

13 Miura H, Ijichi M, Ando Y, et al. A rapidly progressive and fatal case of nonalcoholic steatohepatitis following pancreaticoduodenectomy. Clin J Gastroenterol 2013;6: 470-475.

14 Hocking MP, Duerson MC, O'Leary JP, et al. Jejunoileal bypass for morbid obesity. Late follow-up in 100 cases. N Engl J Med 1983;308:995-999.

15 Cotler SJ, Vitello JM, Guzman G, et al. Hepatic decompensation after gastric bypass surgery for severe obesity. Dig Dis Sci 2004;49:1563-1568.

16 Caldwell SH, Hespenheide EE. Subacute liver failure in obese women. Am J Gastroenterol 2002;97:2058-2062.

17 Shaffer EA. Bariatric surgery: a promising solution for nonalcoholic steatohepatitis in the very obese. J Clin Gastroenterol 2006;40:S44-S50.

18 Mattar SG, Velcu LM, Rabinovitz M, et al. Surgicallyinduced weight loss significantly improves nonalcoholic fatty liver disease and the metabolic syndrome. Ann Surg 2005;242:610-617.

19 Gill RM, Belt P, Wilson L, et al. Centrizonal arteries and microvessels in nonalcoholic steatohepatitis. Am J Surg Pathol 2011;35:1400-1404.

20 Ferrell LD, Greenberg MS. Special stains can distinguish hepatic necrosis with regenerative nodules from cirrhosis. Liver Int 2007;27:681-686.

21 Nakayama $\mathrm{H}$, Itoh $\mathrm{H}$, Kunita $\mathrm{S}$, et al. Presence of perivenular elastic fibers in nonalcoholic steatohepatitis Fibrosis Stage III. Histol Histopathol 2008;23:407-409.

22 Kleiner DE, Brunt EM, Van Natta M, et al. Design and validation of a histological scoring system for nonalcoholic fatty liver disease. Hepatology 2005;41:1313-1321.

23 Williams AL, Hoofnagle JH. Ratio of serum aspartate to alanine aminotransferase in chronic hepatitis. Relationship to cirrhosis. Gastroenterology 1988;95:734-739.

24 Kleiner DE, Berk PD, Hsu JY, et al. Hepatic pathology among patients without known liver disease undergoing bariatric surgery: observations and a perspective from the longitudinal assessment of bariatric surgery (LABS) study. Semin Liver Dis 2014;34:98-107.

25 Verna EC, Berk PD. Role of fatty acids in the pathogenesis of obesity and fatty liver: impact of bariatric surgery. Semin Liver Dis 2008;28:407-426.

26 de Freitas AC, Campos AC, Coelho JC. The impact of bariatric surgery on nonalcoholic fatty liver disease. Curr Opin Clin Nutr Metab Care 2008;11:267-274.

27 Csak T, Ganz M, Pespisa J, et al. Fatty acid and endotoxin activate inflammasomes in mouse hepatocytes that release danger signals to stimulate immune cells. Hepatology 2011;54:133-144.

28 Welsh FK, Farmery SM, MacLennan K, et al. Gut barrier function in malnourished patients. Gut 1998;42: 396-401.

29 Nolan JP. The role of intestinal endotoxin in liver injury: a long and evolving history. Hepatology 2010;52:1829-1835.

30 Ajmera V, Perito ER, Bass NM, et al. Novel plasma biomarkers associated with liver disease severity in adults with nonalcoholic fatty liver disease. Hepatology 2017;65:65-77. 
31 Day CP. NASH-related liver failure: one hit too many? Am J Gastroenterol 2002;97:1872-1874.

32 Day CP, James OF. Steatohepatitis: a tale of two 'hits'? Gastroenterology 1998;114:842-845.

33 Robinson MK, Rustum RR, Chambers EA, et al. Starvation enhances hepatic free radical release following endotoxemia. J Surg Res 1997;69:325-330.

34 van Zutphen T, Ciapaite J, Bloks VW, et al. Malnutrition-associated liver steatosis and ATP depletion is caused by peroxisomal and mitochondrial dysfunction. J hepatol 2016;65:1198-1208.

35 Marsman WA, Wiesner RH, Rodriguez L, et al. Use of fatty donor liver is associated with diminished early patient and graft survival. Transplantation 1996;62:1246-1251.

36 Anstee QM, Seth D, Day CP. Genetic factors that affect risk of alcoholic and nonalcoholic fatty liver disease. Gastroenterology 2016;150:1728-1744.
37 Peters RL. Patterns of hepatic morphology in jejunoileal bypass patients. Am J Clin Nutr 1977;30: 53-57.

38 O'Leary JP. Hepatic complications of jejunoileal bypass. Semin Liver Dis 1983;3:203-215.

39 Sugerman HJ, Kellum JM, DeMaria EJ. Conversion of proximal to distal gastric bypass for failed gastric bypass for superobesity. J Gastrointest Surg 1997;1: $517-524$.

40 Griffen WO Jr, Young VL, Stevenson CC. A prospective comparison of gastric and jejunoileal bypass procedures for morbid obesity. Surg Obes Relat Dis 2005;1: 163-172.

41 Mahawar KK, Parmar C, Graham Y, et al. Monitoring of Liver Function Tests after Roux-en-Y Gastric Bypass: An Examination of Evidence Base. Obes Surg 2016;26: 2516-2522. 